\title{
EFFECT OF LAYERED STRUCTURE ON THE LATTICE HEAT CAPACITY OF THE RARE EARTH MOLYBDATES
}

\author{
A. Feher, P. Stefányi, A. Orendáčová \\ Departmeñt of Experimental Physics, Šafarik University, \\ nám. Febr. vitazstva 9, CS-041 54 Košice, Czechoslovakia
}

\section{E.E. ANDÉrs AND A.I. Zvyagin}

Institute for Low Temperature Physics and Engineering, Academy of Sciences of the Ukrainian SSR, Lenin Prospect 47, SU-310 164 Kharkov, USSR

(Received January 30, 1991)

Temperature dependence of the heat capacity of layered rare earth molybdates was measured in the temperature range from $0.4 \mathrm{~K}$ to $23 \mathrm{~K}$. It is shown that the temperature dependence of the lattice heat capacity can be described by the " $T^{n}$, where $n \neq 3$. It is shown that differently from $\mathrm{KDy}\left(\mathrm{MoO}_{4}\right)_{2}$ and $\mathrm{CsDyEu}\left(\mathrm{MoO}_{4}\right)_{2}$ for $\mathrm{CsDy}\left(\mathrm{MoO}_{4}\right)_{2}$ and $\mathrm{CsGd}\left(\mathrm{MoO}_{4}\right)_{2}$ we obtained the temperature term with $n<3$, which can be connected with the $2 \mathrm{D}$ behaviour, manifested also in the so-called membrane effect.

PACS numbers: 65.40-f, 63.20-e

\section{Introduction}

Layered dimolybdates with structural formula $\mathrm{MR}\left(\mathrm{MoO}_{4}\right)_{2}(\mathrm{M}=\mathrm{Li}, \mathrm{Na}, \mathrm{K}$, $\mathrm{Rb}, \mathrm{Cs}, \ldots ; \mathrm{R}=\mathrm{Ce}, \mathrm{Pr}, \mathrm{Nd}, \ldots$ ) form an unique group of chain-layered structures showing a variety of specific properties, some of which gradually changing with the change of $M$ resp. $R$ elements. The layered structure of these crystals leads to the anharmonism of the lattice vibrations at low temperaturcs, what in connection with the dynamic instability of the lattice may give rise to a number of structural phase transitions. Some of these transitions are accompanied by changes in the Jahn-Teller mechanism of the onset of a structural phase transition. Phase transitions caused by the Jahn-Teller cooperative effect were observed 
e.g. in $\mathrm{KDy}\left(\mathrm{MoO}_{4}\right)_{2}[\mathrm{l}]$. $\mathrm{CsDy}\left(\mathrm{MoO}_{4}\right)_{2}[2], \ldots$ with the consequence that these compounds were excluded from the $\mathrm{MR}\left(\mathrm{MoO}_{4}\right)_{2}$ group and their physical and chemical properties both above and below the phase transition temperature were investigated by various methods, while other materials from the same group often served for comparison purposes.

Recently, the interest to study this group of material was enhanced by the observation of the phase transition into a complex noncolinear magnetically ordered state with strong anisotropic interaction, taking place at temperatures around $1 \mathrm{~K}$ or even lower [4].

Still another topic in the investigation of dimolybdates is the study of low-temperature peculiarities of the phonon spectrum, connected with the quasi-two-dimensionality of the structure. It is known that even quite simple application of the Debye model on the conception on both one-dimensional (1D) and two-dimensional (2D) crystals with linear dispersion law for acoustic branches of the phonon spectrum gives the following lattice contribution to the heat capacity at low temperatures

$$
\begin{array}{cc}
C_{\mathrm{L}} \propto T & \text { for 1D crystal } \\
C_{\mathrm{L}} \propto T^{2} & \text { for 2D crystal. }
\end{array}
$$

Theory of thermal properties of the chain-like and layered crystals was developed by I.M. Lifshits [5, 6], whose calculations of $1 \mathrm{D}$ and 2D crystal heat capacity included the influence of vibrations with a nonlinear dispersion law and who derived the dispersion relations for the long-wave spectrum part of the vibrations of a chain-like or layered crystal as a whole. In addition to the theory of elasticity of strongly anisotropic crystals, this approximation takes into account also the transversal rigidity of the chains or layers, leading to a minus sign of the thermal expansion coefficient within the layers (the so-called membrane effect). These theoretical predictions were experimentally verified on crystals showing large anisotropy (graphit, boron nitride etc.). Complex crystals of the $\mathrm{MR}\left(\mathrm{MoO}_{4}\right)_{2}$ group with a great number of various atoms in a primitive cell show an anisotropy which is about one tenth of that for graphit or boron nitride. However, in contradiction to this, on some $\mathrm{MR}\left(\mathrm{MoO}_{4}\right)_{2}$ compounds the negative coefficient of the thermal expansion in the layer planes was also observed [7] and this, together with the possibility to control the degree of anisotropy by changing the $M$ and $R$ elements, gives good reasons to study the manifestations of the structural low-dimensionality in the heat capacity and enables following considerations on the peculiarities of the phonon spectrum to be made.

\section{Samples and their structure}

We investigated the low-temperature heat capacity on single crystals of layered dimolybdates $\mathrm{CsDy}\left(\mathrm{MoO}_{4}\right)_{2}, \quad \mathrm{KDy}\left(\mathrm{MoO}_{4}\right)_{2}, \mathrm{CsDy}\left(\mathrm{MoO}_{4}\right)_{2}, \quad$ and $\mathrm{CsDy}_{0.95} \mathrm{Eu}_{0.05}\left(\mathrm{MoO}_{4}\right)_{2}$, prepared by the flux method. Cesium resp. potassium form with heavy rare earth elements dimolybdates with rhombic structure at room temperature (space group $D_{2 h}^{3}$ ). Primitive cell parameters for $\operatorname{CsDy}\left(\mathrm{MoO}_{4}\right)_{2}$ are 
$a=0.951 \mathrm{~nm}, b=0.797 \mathrm{~nm}, c=0.505 \mathrm{~nm}$, for $\mathrm{KDy}\left(\mathrm{MoO}_{4}\right)_{2} a=1.821 \mathrm{~nm}$, $b=0.800 \mathrm{~nm}, c=0.510 \mathrm{~nm}$ and for $\operatorname{CsGd}\left(\mathrm{MoO}_{4}\right)_{2} a=0.952 \mathrm{~nm}, b=0.850$ $\mathrm{nm}$, and $c=0.507 \mathrm{~nm}$. In the closest packing plane $b c$ the crystals are easily split into mica-like lamellae. The ac plane is also a plane of close packing. $M$ and $R$ atoms are surrounded by oxygen octahedrons and form chains along the $b$ axis. Translation identical chains of the $M$ resp. $R$ polyhedrons are interconnected by the $\mathrm{MoO}_{4}$ tetrahedrons, whose two peaks link also adjacent polyhedrons within a single chain [8]. In $\mathrm{CsDy}\left(\mathrm{MoO}_{4}\right)_{2}$ the structural phase transition of the I-type with Jahn-Teller mechanism takes place at $42 \mathrm{~K}$, while in $\mathrm{KDy}\left(\mathrm{MoO}_{4}\right)_{2}$ the structural phase transition of the II-type caused by the cooperative Jahn-Teller effect takes place at $14 \mathrm{~K}$. Isomorphous replacement of $\mathrm{Dy}^{3+}$ ions by the $\mathrm{Eu}^{3+}$ ions lowers the temperature of the structural phase transition of the I-type, which then disappears at a concentration of about $5.5 \%$ Eu. $\mathrm{CsGd}\left(\mathrm{MoO}_{4}\right)_{2}$ shows no structural phase transition of the Jahn-Teller character.

Some peculiarities are observed in the phonon spectra of these layered structures. Due to anisotropy of the mutual elastic couplings between layers resp. chains some elastic constant are low leading to low-frequency transversal acoustic modes; dispersion of the longitudinal acoustic mode is, however, very close to that of the three-dimensional crystal. Strong anisotropy of the thermal expansion coefficients $\alpha(T)$ in $\operatorname{CsDy}\left(\mathrm{MoO}_{4}\right)_{2}$, confirmed the existence of the weak inter-layer couplings and was an indication for the existence of the low-frequency phonon branches, really observed later. The ratio of the elastic modulus within layer to that measured between layers was estimated for $\operatorname{CsDy}\left(\mathrm{MoO}_{4}\right)_{2}$ to be close to 10 . It was found that the $\mathrm{Eu}^{3+}$ addition in $\mathrm{CsDy}\left(\mathrm{MoO}_{4}\right)_{2}$ causes the decrease of the elastic modulus within the layer (chain), leading to the change in anisotropy of $\alpha(T)$ and to the disappearance of the minus sign at $\alpha_{b}(T)$ ( $\alpha$ is measured in the $b$ axis direction). Because the ion radius of the alkaline metals at the $M$ site increases with the increasing atomic number, the coupling between layers in the rhombic crystals $\operatorname{MDy}\left(\mathrm{MoO}_{4}\right)_{2}$ is increased with the increase of the alkaline metal ion radius. It can be therefore expected that the low-dimensionality of the structure will be more pronounced in $\mathrm{CsDy}\left(\mathrm{MoO}_{4}\right)_{2}$ than in $\mathrm{KDy}\left(\mathrm{MoO}_{4}\right)_{2}$.

\section{Experimental method}

The heat capacity from $0.4 \mathrm{~K}$ to $23 \mathrm{~K}$ was measured by the method of quasiadiabatic calorimetry. The sample weight varied from $1.2 \mathrm{~g}$ to $2.5 \mathrm{~g}$. Sample temperature was measured by Allen-Bradley carbon resistors, which were ground to plates $0.8 \mathrm{~mm}$ thin and glued to the sample using Varnish GE 7031 to obtain a good thermal contact [9].

\section{Results}

For the evaluation of the heat capacity data the following procedure was used. Since the scheme of the electron energy levels of the magnetic ions is known 
from the EPR measurements; it was possible to calculate the Schottky contribution to the heat capacity $C_{\mathrm{SH}}$ corresponding to the particular energy level scheme. In $\mathrm{CsDy}\left(\mathrm{MoO}_{4}\right)_{2}$ and $\mathrm{CsDyEu}\left(\mathrm{MoO}_{4}\right)_{2}$ crystals with quite large separation between the first excited doublets and the ground doublet, the Schottky heat capacity is negligible in the investigated temperature range. For $\mathrm{KDy}\left(\mathrm{MoO}_{4}\right)_{2}$ and $\mathrm{CsGd}\left(\mathrm{MoO}_{4}\right)_{2}$ samples the Schottky contribution was subtracted from the total observed heat capacity $[10,11]$. Then the temperature range was established, within which the mathematic separation of the lattice $C_{\mathrm{L}}$ and magnetic $C_{\mathrm{M}}$ contribution to the heat capacity could be done with minimum error. Because magnetic contribution to the heat capacity (independently from the origin of the interaction) at temperatures above the phase transition into the magnetically ordered states may be written as $C_{\mathrm{M}} \propto T^{-2}$ and considering that the lattice contribution, in agreement with the Debye theory, is proportional to $T^{3}$ in the low-temperature limit, a temperature range may be found in the materials with the temperature $T_{\mathrm{C}}$ (magnetic phase transition) well below Debye temperature $\theta$, within which the heat capacity may be expressed as

$$
C-C_{\mathrm{SH}}=\beta T^{3}+b T^{-2},
$$

or, after rewriting, as

$$
\left(C-C_{\mathrm{SH}}\right) T^{2}=\beta T^{5}+b,
$$

where $\dot{\beta}$ and $b$ are temperature independent coefficients. Temperature intervals $\left(T_{\min }, T_{\max }\right)$, in which Eq. (2) is valid, were determined for all samples from linear parts of dependences $\left(C-C_{\mathrm{SH}}\right) T^{2}$ vs. $T^{5}$ (Table I).

TABLE I

\begin{tabular}{l|c|c|c|c}
\hline \hline & $T_{\mathrm{C}}$ & \multicolumn{2}{|c|}{ Eq. (2) validity range } & $\theta$ \\
\cline { 3 - 5 } Sample & {$[\mathrm{K}]$} & $T_{\min }[\mathrm{K}]$ & $T_{\max }[\mathrm{K}]$ & {$[\mathrm{K}]$} \\
\hline $\mathrm{CsGd}\left(\mathrm{MoO}_{4}\right)_{2}$ & $0.448 \pm 0.004$ & 2.60 & 4.88 & 158 \\
$\mathrm{KDy}\left(\mathrm{MoO}_{4}\right)_{2}$ & $1.000 \pm 0.005$ & 2.14 & 4.17 & 137 \\
$\mathrm{CsDy}\left(\mathrm{MoO}_{4}\right)_{2}$ & $1.294 \pm 0.005$ & 2.70 & 5.10 & 171 \\
$\mathrm{CsDyEu}\left(\mathrm{MoO}_{4}\right)_{2}$ & $1.226 \pm 0.005$ & 3.18 & 4.70 & 161
\end{tabular}

In the so-determined validity range the coefficients of both magnetic $(b)$ and lattice $(\beta)$ contributions were determined from the observed heat capacity, using the least square method. The value of $\beta$ was then put into the formula:

$$
\theta=(1944 n \beta)^{1 / 3},
$$

where $n$ is the effective number of vibrating groups in a primitive cell, and so the effective values of the so-called Debye temperatures $\theta$ (see Table I), characterizing the crystal bonding forces, were calculated.

The term $b T^{-2}$ representing magnetic contribution was subtracted from the heat capacity $\left(C-C_{\mathrm{SH}}\right)$ for $T>T_{\min }$ and thus the resulting lattice contribution $C_{\mathrm{L}}$ was further studied. However, above $T_{\max }$ the formula:

$$
C_{L}=\beta T^{3} \text {. }
$$


is no more a good fit for the experimentally observed $C_{\mathrm{L}}(T)$ behaviour and the lattice contributions to the heat capacity should be written in the form:

$$
C_{L}=\beta^{\prime} T^{n} \quad \text { and } n \neq 3,
$$

where $\beta^{\prime}$ is a coefficient independent of temperature. The presence of $T^{n}$ term in Eq. (4) for $n \neq 3$ in $C_{\mathrm{L}}$ may be observed in $\left(C-C_{\mathrm{SH}}-C_{\mathrm{M}}\right) / T^{3}$ vs. $T^{2}$, where any deviation from the dependence (4) is detected as deviation from the straight line parallel to $x$ (see Fig. 1). It may be shown that when the experimental heat capacity

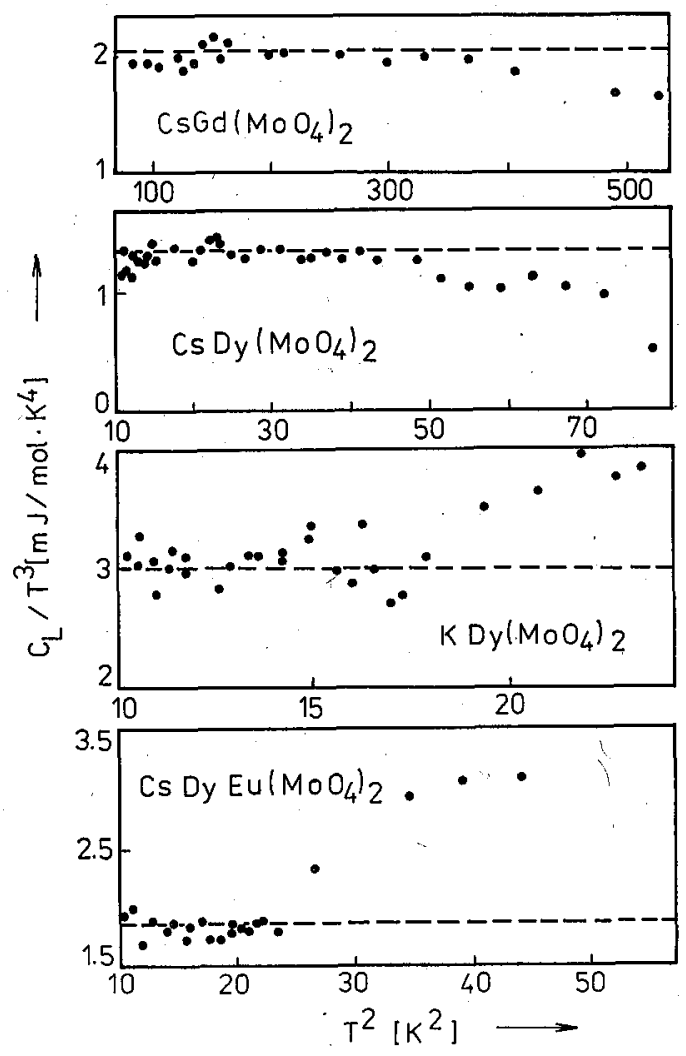

Fig. 1. The lattice heat capacity of investigated samples.

dependence deviates from the horizontal line in "up" direction, term with higher $n(n>3)$ will appear in $C_{L}$, and vice versa, the "down" deviation corresponds to the presence of term with lower $n(n<3)$. As is seen from Fig. 1, the investigated samples have no uniform form of $C_{\mathrm{L}}(T)$ for $T>T_{\max }$. The $C_{\mathrm{L}}(T)$ deviation in $\mathrm{CsGd}\left(\mathrm{MoO}_{4}\right)_{2}$ and $\mathrm{CsDy}\left(\mathrm{MoO}_{4}\right)_{2}$ samples in the "down" direction indicates the presence of low-power temperature term $n<3$. The "up" deviation of the heat capacity in the $\mathrm{KDy}\left(\mathrm{MoO}_{4}\right)_{2}$ and $\mathrm{CsDy}\left(\mathrm{MoO}_{4}\right)_{2}$ samples indicates the presence of $T^{n}$ term with $n>3$. 


\section{Discussion}

Our results on $\mathrm{CsGd}\left(\mathrm{MoO}_{4}\right)_{2}$ and $\mathrm{CsDy}\left(\mathrm{MoO}_{4}\right)_{2}$ are in agreement with [6], where the existence of the $T^{2}$ term is expected for layered crystals, what was confirmed by the results of $[12,13]$ indicating low-dimensionality in $\mathrm{CsDy}\left(\mathrm{MoO}_{4}\right)_{2}$. In the other two samples the presence of higher $T^{n}$ power $(n>3)$ indicates that the manifestations of the low-dimensionality will be weakened in these crystals, whose properties will then approach those found in 3D crystals. For $\mathrm{KDy}\left(\mathrm{MOO}_{4}\right)_{2}$ such a behaviour is supported by results of [13], where the influence of the ion radii of alkaline metals $M$ on the structural low-dimensionality were studied with the conclusion that with the increase of the ion radius, by going from $\mathrm{K}$ to $\mathrm{Cs}$, the 2D behaviour of the material is "improved". A very interesting results were obtained for $\mathrm{CsDyEu}\left(\mathrm{MoO}_{4}\right)_{2}$, where the replacement of only $5 \%$ of $\mathrm{Dy}^{3+}$ ions by the $\mathrm{Eu}^{3+}$ ions leads to a radical change in the crystal behaviour, "increasing" its dimensionality so that the properties of this crystal decline from the properties of the pure $\mathrm{CsDy}\left(\mathrm{MoO}_{4}\right)_{2}$ and approach those of $\mathrm{KDy}\left(\mathrm{MoO}_{4}\right)_{2}$. This effect may be put in connection with the observations in [12], that at such a concentration of $\mathrm{Eu}$ the structural phase transition of the I-type is replaced by the phase transition of the II-type (similarly as in $\left.\mathrm{KDy}\left(\mathrm{MoO}_{4}\right)_{2}\right)$ and with [14], where the change of magnetic properties was observed at the concentration of $5 \% \mathrm{Eu}^{3+}$. According to the authors of [14], the observed change in the magnetic behaviour may be connected with the fact that the structural phase transition of the I-type is replaced by the phase transition of the II-type.

We can conclude that terms characteristic of the structural 2D systems were found in the heat capacity of $\mathrm{CsGd}\left(\mathrm{MoO}_{4}\right)_{2}$ and $\mathrm{CsDy}\left(\mathrm{MoO}_{4}\right)_{2}$ samples, while the heat capacity of $\mathrm{KDy}\left(\mathrm{MoO}_{4}\right)_{2}$ and $\mathrm{CsDyEu}\left(\mathrm{MoO}_{4}\right)_{2}$ shows indications typical for the structural 3D systems. It may be expected that in $\mathrm{CsGd}\left(\mathrm{MoO}_{4}\right)_{2}$ and $\mathrm{CsDy}\left(\mathrm{MoO}_{4}\right)_{2}$ crystals the long-wave part of the phonon spectrum would contain a quadratic dispersion law predicted by Lifshits and this may have an apparent influence also on the magnetic properties of the investigated samples.

\section{References}

[1] A.I. Zvyagin, T.S. Stetsenko, V.G. Yurko, R.A. Vaishnoras, Pis'ma Zh. Eksp. Teor. Fiz. 17, 190 (1973).

[2] A.I. Zvyagin, S.D. Elchaninova, T.S. Stetsenko, L.N. Pelich, E.N. Khatsko, Sov. J. Low Temp. Phys. 1, 79'(1975).

[3] A.I. Zvyagin, T.S. Stetsenko, V.G. Yurko, R.A. Vaishnoras, Pis'ma Zh. Eksp. Teor. Fiz. 17, 135 (1973).

[4] P. Stefányi, A. Feher, A. Orendáčová, E.E. Anders, A.I. Zvyagin, J. Phys. (France) 50, 1297 (1989).

[5] I.M. Lifshits, Zh. Eksp. Teor. Fiz. 22, 471 (1952).

[6] I.M. Lifshits, Zh. Eksp. Teor. Fiz. 22, 475 (1952).

[7] S.B. Feodosiev, E.S. Syrkin, I.A. Gospodarev, V.P. Popov, A.A. Gurskas, N.M. Nesterenko, Sov. Phys.-Solid State 31, 186 (1989). 
[8] A.V. Vinokurov, P.V. Klevtsov, Kristállografiya 17, 127 (1972).

[9] P. Skyba, V. Makroczyová, P. Stefányi, A. Feher, P. Petrovič, Čs. Cas. Fyz. A 38, 371 (1988).

[10] P. Stefányi, A. Feher, A. Orendáčová, J. Phys., Condens. Matter 1, 7529 (1989).

[11] A. Feher, P. Stefányi, A. Orendáčová, E.E. Anders, A.G. Anders, S.V. Volotskii, A.I. Zvyagin, S.V. Startsev, E.N. Khatsko, A.S. Cherny, Sov. Phys.-Low Temp. 14, 1305 (1988).

[12] S.D. Elchaninova, A.S. Ilyushin, Yu.G. Litvinenko, Sov. Phys.-Low Temp. 12, 616 (1986).

[13] L.N. Pelich, A.A. Gurskas, A.I. Zvyagin, in: Proc. 2lst Soviet Low Temp. Conference, ed. E.N. Khatsko, Kharkov 1980, p. 12.

[14] E.N. Khatsko, A.S. Cherny, in Proc. 23rd Soviet Low Temp. Conference, ed. V. Kozovits, Tallin 1984, p. 50. 